\title{
Generalized entropies and corresponding holographic dark energy models
}

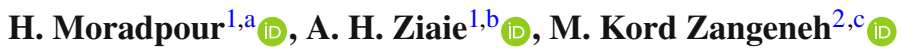 \\ ${ }^{1}$ Research Institute for Astronomy and Astrophysics of Maragha (RIAAM), University of Maragheh, P.O. Box 55136-553, Maragheh, Iran \\ ${ }^{2}$ Physics Department, Faculty of Science, Shahid Chamran University of Ahvaz, Ahvaz 61357-43135, Iran
}

Received: 29 April 2020 / Accepted: 31 July 2020 / Published online: 12 August 2020

(C) The Author(s) 2020

\begin{abstract}
Using Tsallis statistics and its relation with Boltzmann entropy, the Tsallis entropy content of black holes is achieved, a result in full agreement with a recent study (Mejrhit and Ennadifi in Phys Lett B 794:24, 2019). In addition, employing Kaniadakis statistics and its relation with that of Tsallis, the Kaniadakis entropy of black holes is obtained. The Sharma-Mittal and Rényi entropy contents of black holes are also addressed by employing their relations with Tsallis entropy. Thereinafter, relying on the holographic dark energy hypothesis and the obtained entropies, two new holographic dark energy models are introduced and their implications on the dynamics of a flat FRW universe are studied when there is also a pressureless fluid in background. In our setup, the apparent horizon is considered as the IR cutoff, and there is not any mutual interaction between the cosmic fluids. The results indicate that the obtained cosmological models have (i) notable powers to describe the cosmic evolution from the matter-dominated era to the current accelerating universe, and (ii) suitable predictions for the universe age.
\end{abstract}

\section{Introduction}

Originally, Gibbs put forth that systems including long-range interactions may not be extensive [1], a hypothesis which also motivates people to propose various entropy definitions [24]. Recently, such entropies have been employed to model the cosmic evolution in various setups [5-9]. Generalized entropies have also been employed to study black holes [1016] and also to build new holographic dark energy models [17-19]. Additionally, it has been shown that such entropies (i) can provide a theoretical basis for the MOND theory [20], (ii) affect the Jeans mass [21], (iii) may be motivated by

\footnotetext{
a e-mail: hn.moradpour@maragheh.ac.ir (corresponding author)

be-mail: ah.ziaie@maragheh.ac.ir

ce-mail: mkzangeneh@scu.ac.ir
}

the quantum features of gravity [22,23], and even, (iv) may describe inflation without considering inflaton [24].

Bekenstein entropy [25] (and therefore, the nature of degrees of freedom of horizon [26,27]) is the cornerstone of primary holographic dark energy hypothesis (PHDE) [28], a promising approach to understand the origin of dark energy. While apparent horizon is a proper causal boundary for cosmos meeting conservation and thermodynamics laws [3035], PHDE with apparent horizon as IR cutoff suffers from some weaknesses $[28,29]$. On the other, three generalized entropy based holographic dark energy models have been proposed that can provide considerable descriptions for the universe expansion even if apparent horizon is employed as IR cutoff [17-19,36]. Therefore, the use of generalized entropies may help us in finding more suitable models of HDE.

Generalized entropies should obey fundamental laws such as zero law of thermodynamics and some pioneering arguments on the zeroth law compatibility of them can be found in Refs. [37-44]. Recently, some cosmological and gravitational consequences of Kaniadakis statistics [6,45], as a generalized entropy measure [2-4], have been studied. Here, motivated by the above arguments, we are going to (i) calculate the entropy of black holes in the various well-known generalized entropy formalisms, and (ii) study their ability in describing the current accelerated universe by building their corresponding holographic dark energy models in the unit of $k_{B}=G=c=\hbar=1$.

In the next section, after addressing relation between Tsallis and Boltzmann entropies, Tsallis entropy of black holes is derived, a result compatible with that of Ref. [46]. In addition, having introduced Kaniadakis entropy and its relation with Tsallis entropy, we also compute the Kaniadakis entropy of black hole in the next section. Focusing on the relations between Tsallis entropy and Sharma-Mittal and Rényi entropies [18,19], the Sharma-Mittal and Rényi entropy contents of black holes are other issues which will 
also be addressed in the second section. The Kaniadakis holographic dark energy (KHDE) shall be introduced in Sect. 3, where some of its cosmological consequences are also investigated. The ability of obtained Tsallis entropy in describing dark energy as the vacuum energy, through constructing the corresponding HDE model, is also studied in Sect. 4. The universe age is finally addressed in the fifth section. In the last section, some concluding remarks have been collected.

\section{Tsallis and Kaniadakis entropies of black holes}

Working in the unit $k_{B}=1$, both the Shannon and Gibbs entropies of a distribution with $W$ states lead to the same expression

$S=-\sum_{i=1}^{W} P_{i} \ln \left(P_{i}\right)$

while $P_{i}$ denotes the probability of occupying the $i$ th state, for the classical systems. The quantum mechanical version of this entropy, the so-called Von-Neumann entropy, is also presented as

$S=-\operatorname{Tr}[\rho \ln (\rho)]$.

The use of Eq. (2) for classical systems goes indeed back to the Boltzmann's proposal where $\rho$ is the state density in phase space [47].

Applying Eq. (2) to a purely gravitational system, the socalled Bekenstein entropy ( $\equiv S_{B H}=\frac{A}{4}$ ) is obtainable [25]. Since the degrees of freedom are distributed on horizon without any specific priority with respect to one another, one may assume that, at least based on our knowledge [26,27], $P_{i}$ is equal for all of them allowing us in writing $P_{i}=\frac{1}{W}$. In this manner, both of the above relations lead to the Boltzmann entropy $(S=\ln (W))$, and hence, we have $[9,45]$

$S_{B H}=\frac{A}{4}=\ln (W) \rightarrow W=\exp \left(\frac{A}{4}\right)$,

for the horizon entropy, and consequently $W(A)$.

The Tsallis entropy, as a single-free parameter generalized entropy, is defined as [4]

$S_{Q}^{T}=\frac{1}{1-Q} \sum_{i=1}^{W}\left(P_{i}^{Q}-P_{i}\right)=\frac{W^{1-Q}-1}{1-Q}$,

where $Q$, named Tsallis or non-extensive parameter, is an unknown free parameter $\left(S_{Q}^{T} \rightarrow S\right.$ for $\left.Q \rightarrow 1\right)$, and the last line is valid only for probability distributions meeting the $P_{i}=\frac{1}{W}$ condition. The $Q$ parameter may also be arisen from the quantum features of gravity $[22,45]$. Now, using Eq. (3) and the last line of Eq. (4), and defining $\delta=1-Q$, one can easily find

$$
\begin{aligned}
S_{Q}^{T} & =\frac{1}{1-Q}\left[\exp \left((1-Q) S_{B H}\right)-1\right] \\
& =\frac{2 \exp \left(\frac{\delta S_{B H}}{2}\right)}{\delta} \sinh \left(\frac{\delta S_{B H}}{2}\right) .
\end{aligned}
$$

In the loop quantum gravity scenario, by applying Tsallis entropy definition to black holes, it is obtained that [46]

$S_{Q}^{T}=\frac{1}{1-Q}\left[\exp \left(\frac{(1-Q) \ln (2)}{\gamma \pi \sqrt{3}} S_{B H}\right)-1\right]$,

satisfying the $S_{Q}^{T} \rightarrow S_{B H}$ expectation whenever $Q \rightarrow 1$ and $\gamma=\frac{\ln (2)}{\pi \sqrt{3}}$ [46]. Therefore, in order to preserve the $S_{Q}^{T} \rightarrow$ $S_{B H}$ expectation at the $Q \rightarrow 1$ limit, we consider $\gamma=\frac{\ln (2)}{\pi \sqrt{3}}$, and accordingly, Eqs. (6) and (5) become the same.

The Kaniadakis entropy ( $\kappa$-entropy), as another singlefree parameter generalized entropy, is also defined as [2,3]

$$
\begin{aligned}
S_{\kappa} & =-\sum_{i=1}^{W} \frac{P_{i}^{1+\kappa}-P_{i}^{1-\kappa}}{2 \kappa} \\
& =\frac{1}{2}\left(\frac{\sum_{i=1}^{W}\left(P_{i}^{1-\kappa}-P_{i}\right)}{\kappa}+\frac{\sum_{i=1}^{W}\left(P_{i}^{1+\kappa}-P_{i}\right)}{-\kappa}\right),
\end{aligned}
$$

where $\kappa$ is an unknown parameter, and the Boltzmann-Gibbs entropy is recovered at the $\kappa \rightarrow 0$ limit $[2,3]$. Comparing Eqs. (7) and (4) with each other, one can easily obtain

$S_{\kappa}=\frac{S_{1+\kappa}^{T}+S_{1-\kappa}^{T}}{2}$.

Moreover, by assuming $P_{i}=\frac{1}{W}$, Eq. (7) helps us in getting $[2,3]$

$S_{\kappa}=\frac{W^{\kappa}-W^{-\kappa}}{2 \kappa}$,

combined with Eq. (3) to reach at

$S_{\kappa}=\frac{1}{\kappa} \sinh \left(\kappa S_{B H}\right)$.

Indeed, putting Eq. (6) or Eq. (5) in Eq. (8), one can again reach this result, the Kaniadakis entropy of a black hole.

Now, as a brief study, let us investigate the SharmaMittal and Rényi entropy contents of a black hole with Tsallis entropy (5). Since Sharma-Mittal $\left(S_{S M}\right)$ and Rényi $(\mathcal{S})$ entropies can be expressed as the functions of Tsallis entropy as $[18,19]$

$$
\begin{aligned}
S_{S M} & =\frac{1}{R}\left(\left(1+(1-Q) S_{T}\right)^{\frac{R}{1-Q}}-1\right) \\
\mathcal{S} & =\frac{1}{1-Q} \ln \left(1+(1-Q) S_{T}\right)
\end{aligned}
$$


respectively, in which $R$ is an unknown parameter [18,19], a straightforward calculation leads to

$$
\begin{aligned}
S_{S M} & =\frac{1}{R}\left[\exp \left(R S_{B H}\right)-1\right], \\
\mathcal{S} & =S_{B H},
\end{aligned}
$$

where we assumed $\gamma=\frac{\ln (2)}{\pi \sqrt{3}}$, otherwise $\frac{\ln (2)}{\gamma \pi \sqrt{3}} S_{B H}$ would emerge in results instead of $S_{B H}$. The obtained $S_{S M}$ and $\mathcal{S}$ are mathematically equal to Tsallis entropy (5) and Bekenstein entropy, respectively, meaning that they can not tell us anything more than what Tsallis entropy (5) and Bekenstein entropy give us in constructing HDE models. Hence, we only focus on Eqs. (10) and (5) to address two new HDE models.

\section{KHDE}

Bearing the Kaniadakis entropy content of a black hole in mind (10), since the HDE hypothesis claims that if vacuum energy handles the current accelerated universe, then its amount stored in a box with size $L^{3}$ should not exceed the energy of its same size black hole [28], one can reach

$\Lambda^{4} \equiv \rho_{\Lambda}^{\kappa} \propto \frac{S_{\kappa}}{L^{4}}$,

for the vacuum energy $\rho_{\Lambda}^{\kappa}$. Now, considering the apparent horizon of flat FRW universe as the IR cutoff (i.e. $L=\frac{1}{H} \Rightarrow A=\frac{4 \pi}{H^{2}}$ ), we find

$\rho_{\Lambda}^{\kappa}=\frac{3 C^{2} H^{4}}{8 \pi \kappa} \sinh \left(\frac{\pi \kappa}{H^{2}}\right)$,

where $C^{2}$ is an unknown constant as usual [28]. Now, it is apparent that whenever $\kappa \rightarrow 0$, we have $\rho_{\Lambda}^{\kappa} \rightarrow \frac{3 C^{2} H^{2}}{8 \pi}$, the well-known Bekenstein entropy-based HDE (i.e. PHDE) [28]. In the absence of a mutual interaction between the cosmic sectors, including a pressureless fluid with energy density $\rho_{m}$ and the dark energy candidate with energy density $\rho_{\Lambda}^{\kappa}$ and pressure $p_{\Lambda}$, the energy-momentum conservation law and Friedmann equations take the forms

$$
\begin{aligned}
& \dot{\rho}_{m}+3 H \rho_{m}=0, \\
& p_{\Lambda}^{\kappa}=-\left(\frac{\dot{\rho}_{\Lambda}^{\kappa}}{3 H}+\rho_{\Lambda}^{\kappa}\right),
\end{aligned}
$$

and

$$
\begin{aligned}
& H^{2}=\frac{8 \pi}{3}\left(\rho_{m}+\rho_{\Lambda}^{\kappa}\right), \\
& H^{2}+\frac{2}{3} \dot{H}=\frac{-8 \pi}{3}\left(p_{\Lambda}^{\kappa}\right),
\end{aligned}
$$

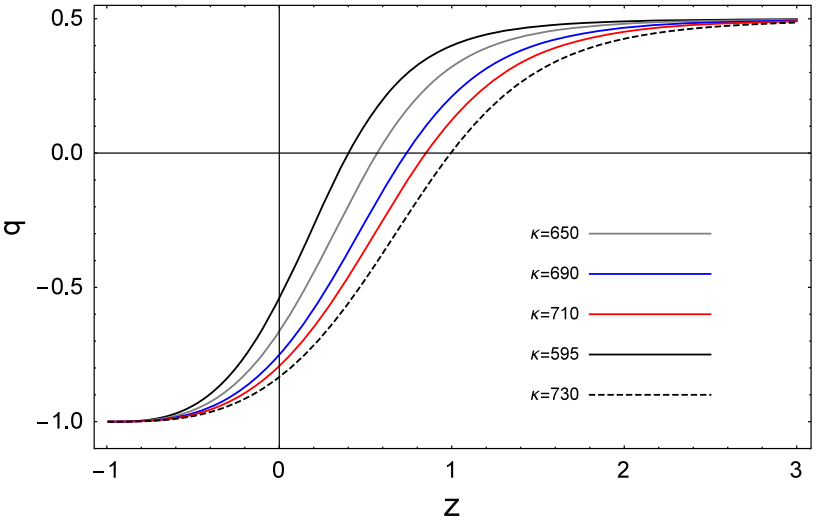

Fig. 1 The behavior of deceleration parameter for $C^{2}=0.3025$, $\Omega_{m}^{0}=0.315$ (the current value of $\Omega_{m}$ ), and $H_{0}=67.9$ [48]

respectively, where dot denotes derivative with respect to time. Deceleration parameter $q$ is also obtainable as

$q=-1-\frac{\dot{H}}{H^{2}}=\frac{1}{2}\left(1+\frac{3 \omega_{\Lambda}^{\kappa}}{\frac{\Omega_{m}}{\Omega_{\Lambda}^{\kappa}}+1}\right)$,

in which

$$
\begin{aligned}
& \Omega_{\Lambda}^{\kappa} \equiv \frac{\rho_{\Lambda}^{\kappa}}{\rho_{c}}=\frac{C^{2} H^{2}}{\kappa} \sinh \left(\frac{\pi \kappa}{H^{2}}\right), \\
& \Omega_{m} \equiv \frac{\rho_{m}}{\rho_{c}}, \omega_{\Lambda}^{\kappa} \equiv \frac{p_{\Lambda}^{\kappa}}{\rho_{\Lambda}^{\kappa}}, \rho_{c}=\frac{3 H^{2}}{8 \pi} .
\end{aligned}
$$

Evolution of $q$ versus redshift $z$ has been plotted in Fig. 1, which clearly shows that, depending on the values of $C^{2}$ and $\kappa$, a desired behavior is getable. These values can also affect the value of transition redshift $z_{t r}$ at which $q=0$ and universe changes its acceleration phase.

$w_{\Lambda}^{\kappa}$ is also depicted as a function of $q$ only for $\kappa=690$ in Fig. 2, because the curves corresponding to other values of $\kappa$, used in plotting Fig. 1, are so close to this curve. As it is apparent, KHDE behaves as a pressureless fluid for high redshift limits (when $q=\frac{1}{2}$ ) and mimics a cosmological constant $\left(\equiv w_{\Lambda}^{\kappa} \rightarrow-1\right)$ when $z \rightarrow-1$ for which $q \rightarrow-1$ and $\rho_{m} \rightarrow 0$.

In fact, by decreasing $z$, KHDE starts to control the universe expansion rate and approaches its maximum (1) when $z \rightarrow-1$ or equally $q \rightarrow-1$. At high redshift limits, the energy density of matter density begins to become dominant and matter dominated era is begun to be covered i.e. we have $q \rightarrow \frac{1}{2}$ and $\frac{\Omega_{\Lambda}^{\kappa}}{\Omega_{m}} \rightarrow 0$. In order to have a better look, we plotted $q\left(\frac{\Omega_{m}}{\Omega_{\Lambda}^{k}}\right)$ in Fig. 3. As it is apparent, the ratio $\frac{\Omega_{m}}{\Omega_{\Lambda}^{\kappa}}$ increases as a function of $z$, and the matter dominated era $\left(q=\frac{1}{2}\right)$ shall be recovered at high redshift limit in full agreement with what one observes in previous figures. At the transition point, we have $q=0$ leading to 


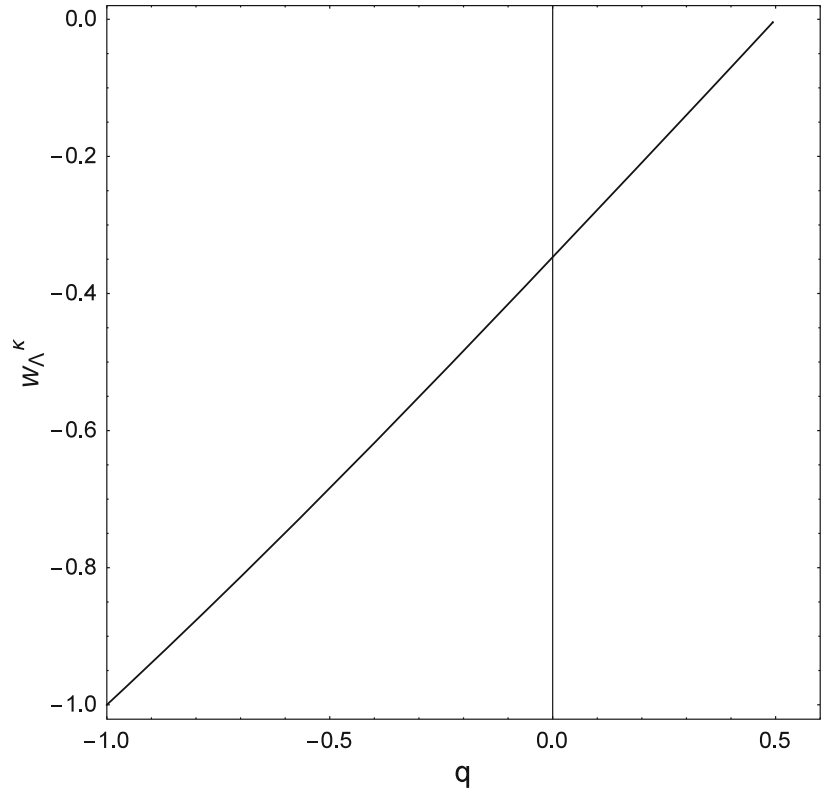

Fig. 2 The behavior of $w_{\Lambda}^{\kappa}$ against deceleration parameter for $C^{2}=$ 0.3025, $\Omega_{m}^{0}=0.315, H_{0}=67.9$ and $\kappa=690$

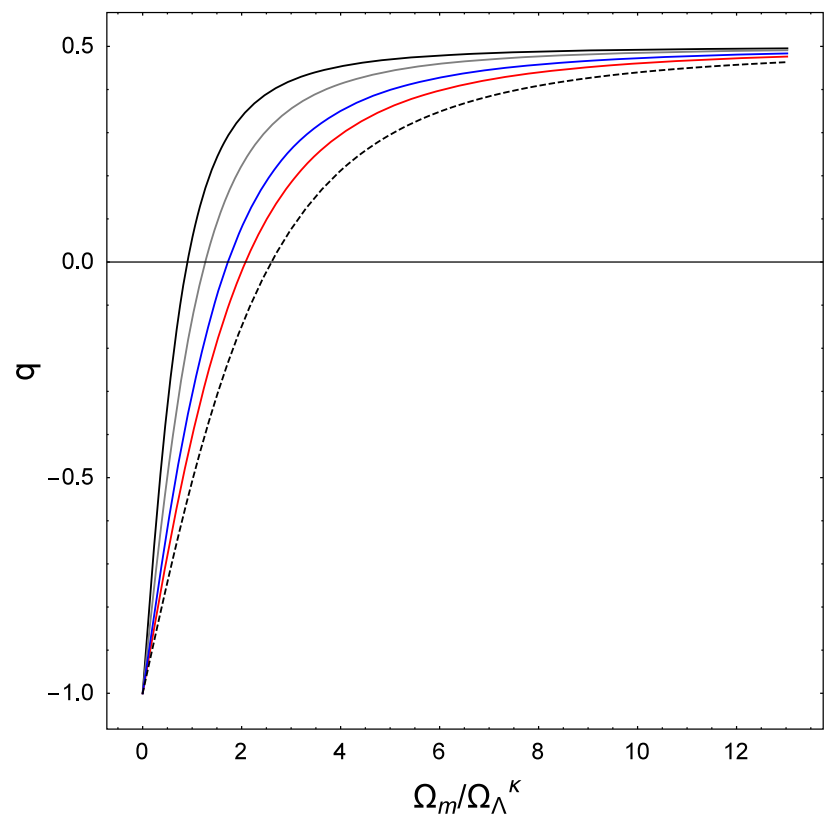

Fig. 3 The behavior of deceleration parameter against the ratio $\Omega_{m} / \Omega_{\Lambda}^{\kappa}$ for $C^{2}=0.3025, \Omega_{m}^{0}=0.315, H_{0}=67.9$. We have $\Omega_{m} / \Omega_{\Lambda}^{\kappa} \approx 0.95$ at $z_{t r} \approx 0.570$ for gray curve and $\Omega_{m} / \Omega_{\Lambda}^{\kappa} \approx 2.01$ at $z_{t r} \approx 0.997$ for black dashed curve

$\frac{\Omega_{m}}{\Omega_{\Lambda}^{\kappa}}=-1-3 \omega_{\Lambda}^{\kappa}$,

which states that $\frac{\Omega_{m}}{\Omega_{\Lambda}^{\kappa}}>0$ only if $\omega_{\Lambda}<-\frac{1}{3}$. For example, for $\Lambda$ CDM model, we have $\frac{\Omega_{m}}{\Omega_{\Lambda}^{\kappa}}=2$. In Fig. 3, a variety of the



Fig. 4 The behavior of deceleration parameter for $D^{2}=0.3136$, $\Omega_{m}^{0}=0.315$ (the current value of $\Omega_{m}$ ), and $H_{0}=67.9$

$\frac{\Omega_{m}}{\Omega^{\kappa}}$ ratio is obtainable, depending on the values of $C^{2}$ and $\kappa$ parameters.

\section{New Tsallis Holographic dark energy}

Following the above recipe led to Eqs. (13) and (14) and also by using Tsallis entropy (5) instead of Kaniadakis entropy, one reaches the new Tsallis Holographic dark energy (NTHDE) as

$\rho_{\Lambda}^{T} \propto \frac{S_{Q}^{T}}{L^{4}} \Rightarrow \rho_{\Lambda}^{T}=\left(\frac{3 T^{2}}{8 \pi}\right) \frac{S_{Q}^{T}}{L^{4}}$,

in which $T^{2}$ is an unknown constant as usual [28]. Now, let us define a new constant $D^{2}=\pi T^{2}$ and consider apparent horizon as the IR cutoff ( $L=\frac{1}{H}$ ). Simple calculations lead to

$\rho_{\Lambda}^{T}=\frac{2 D^{2} \rho_{c}}{X} \exp \left(\frac{X}{2}\right) \sinh \left(\frac{X}{2}\right)$,

where $X=\frac{\delta \pi}{H^{2}}$. In this manner, Eqs. (15)-(18) are still valid if we apply the below changes to them

$$
\begin{aligned}
& \rho_{\Lambda}^{\kappa} \Rightarrow \rho_{\Lambda}^{T}, p_{\Lambda}^{\kappa} \Rightarrow p_{\Lambda}^{T}, \omega_{\Lambda}^{\kappa} \Rightarrow \omega_{\Lambda}^{T} \equiv \frac{p_{\Lambda}^{T}}{\rho_{\Lambda}^{T}} \\
& \Omega_{\Lambda}^{\kappa} \Rightarrow \Omega_{\Lambda}^{T} \equiv \frac{\rho_{\Lambda}^{T}}{\rho_{c}}=\frac{2 D^{2}}{X} \exp \left(\frac{X}{2}\right) \sinh \left(\frac{X}{2}\right) .
\end{aligned}
$$

In Figs. 4, 5, deceleration parameter is plotted against redshift and ratio $\frac{\Omega_{m}}{\Omega_{\Lambda}^{T}}$, respectively, indicating that this ratio increases as a function of $z$ and matter dominated era is achievable by increasing redshift. The same as KHDE, NTHDE behaves as pressureless source when $q=\frac{1}{2}$ and mimics cosmological constant $\left(w_{\Lambda}^{T}=-1\right)$ for $q \rightarrow-1$. We also plotted $w_{\Lambda}^{T}$ against $q$ only for $\delta=947$, because of the 




Fig. 5 The behavior of deceleration parameter against the ratio $\Omega_{m} / \Omega_{\Lambda}^{T}$ for $D^{2}=0.3136, \Omega_{m}^{0}=0.315$, and $H_{0}=67.9$

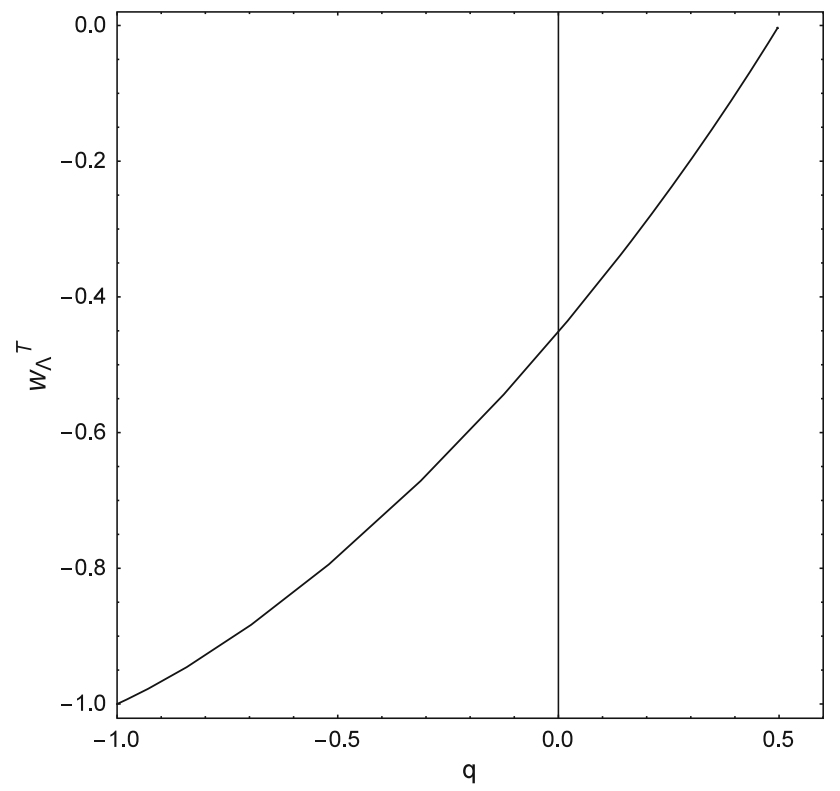

Fig. 6 The behavior of $w_{\Lambda}^{T}$ against deceleration parameter for $D^{2}=$ $0.3136, \Omega_{m}^{0}=0.315, H_{0}=67.9$ and $\delta=947$

proximity of the curves corresponding on other values of $\delta$ to each other, a property also seen in the case of KHDE.

\section{The Universe age}

In order to find the predictions of the above models for the universe age, one can write

$t=\int \frac{d t d H}{d H}=\int \frac{d H}{\dot{H}}=-\frac{1}{4 \pi} \int \frac{d H}{\frac{3 H^{2}}{8 \pi}+p_{\Lambda}}$,

where $p_{\Lambda}$ denotes the pressure of the introduced HDE models, i.e. KHDE and NTHDE. As it is apparent from the behavior of KHDE and NTHDE, when $-1 \leq z$, we have $0<$ $\rho_{\Lambda}<\rho_{c}$ and also $-1 \leq w_{\Lambda} \leq 0 \Rightarrow-p_{\Lambda}=-w_{\Lambda} \rho_{\Lambda} \leq \rho_{\Lambda}$ in which $w_{\Lambda}=w_{\Lambda}^{\kappa}, w_{\Lambda}^{T}$. In this regard, one may estimate Eq. (23) as

$t_{u b} \approx \frac{-2}{3\left(1+w_{\Lambda} \Omega_{\Lambda}\right)_{z=0}} \int \frac{d H}{H^{2}}=\frac{2}{3 H_{0}}\left(1+w_{\Lambda} \Omega_{\Lambda}\right)_{z=0}^{-1}$.

Indeed, for the models, the corresponding pressure approaches zero at high redshift limit and takes negative values by decreasing $z$. It means that the $\frac{1}{\frac{3 H^{2}}{8 \pi}+p_{\Lambda}}$ term always increases during the cosmic evolution. Therefore, in order to reach the above primary estimation, we replaced this term with its current value (i.e. $\left.\left(1+w_{\Lambda} \Omega_{\Lambda}\right)_{z=0}\right)$ and pull it outside the integral. By doing so, an upper bound, depending on the values of model parameters, for the age of current universe $\left(t_{u b}\right)$ can be achieved. The same approximation has also been used in order to guess the universe age in other models $[17,36]$.

Now, let us consider KHDE, for which, depending on the values of $\kappa$ used to plot the curves, we have $-0.925<$ $w_{\Lambda}^{\kappa}(z=0)<-0.74$ when $C^{2}=0.3025$. In this manner, one finds that the upper bound of the universe age is in the range of $\left(\frac{2}{3 H_{0}}<\right) \frac{1.35}{H_{0}}<t_{u b}^{\kappa}<\frac{1.82}{H_{0}}$. For the second case, one reaches $-0.93<w_{\Lambda}^{T}(z=0)<-0.76$ and hence, $\frac{1.39}{H_{0}}<t_{u b}^{T}<\frac{1.84}{H_{0}}$ as the allowed range for the upper bound of the universe age when $\delta$ lies within the range employed to plot the corresponding curves in previous section and $D^{2}=0.3136$. Since we used $\Omega_{m}^{0}=0.315$ in order to plot the diagrams in our investigation, we have $\Omega_{\Lambda}^{0}=1-\Omega_{m}^{0}=0.685$ for the current value of density parameter of dark energy candidate in the models. In both models, the obtained upper bound of the universe age also increases as the current value of state parameter decreases.

On the other hand, during the cosmic evolution, $p_{\Lambda} \leq 0$ and its maximum value (zero) is achieved at high redshift limit. Therefore, at each point $H$, we have $\frac{1}{\frac{3 H^{2}}{8 \pi}} \leq \frac{1}{\frac{3 H^{2}}{8 \pi}+p_{\Lambda}}$ meaning that if one estimates $\frac{1}{\frac{3 H^{2}}{8 \pi}+p_{\Lambda}}$ with $\frac{1 H^{8 \pi}}{\frac{3 H^{2}}{8 \pi}}$, then Eq. (23) gives

$t_{l b} \approx-\frac{2}{3} \int \frac{d H}{H^{2}}=\frac{2}{3 H_{0}}<-\frac{1}{4 \pi} \int \frac{d H}{\frac{3 H^{2}}{8 \pi}+p_{\Lambda}}$, 
indeed, a lower bound on the universe age in the models. In summary, as the predictions of models about the universe age lie within the range $t_{l b}<t<t_{u b}$. Although the lower bound $t_{l b}$ is common in the models, the value of $t_{u b}$ for each case is different, it depends on the values of the models parameters and for the parameters values addressed in the previous sections, it is greater than the Hubble time $t_{H}$ (i.e. $\left.t_{H}=\frac{1}{H_{0}}<t_{u b}\right)$.

It seems that although the age of HD140283 $\left(t_{H D}\right)$ is very close to the Hubble time, due to the accuracy of current measurements, it is still defensible in the framework of the Planck data which predicts a universe younger than $\frac{1}{H_{0}}[48,49]$. In fact, the Planck estimation of the universe age $\left(t_{p}\right)$ is something about $\frac{0.95}{H_{0}}$ [48]. Therefore, $t_{l b}<t_{p} \sim t_{H} \approx t_{H D}<t_{u b}$ meaning that, depending on the values of the models parameters, the models can be free of the age problem.

\section{Concluding remarks}

Relying on relation between Tsallis and Boltzmann entropies and assuming that all degrees of freedom of horizon have same probability, we could obtain the Tsallis entropy of black holes, a result fully compatible with that of Ref. [46]. Thereafter, using the Kaniadakis statistics and its relation with Tsallis statistics, we got the Kaniadakis entropy content of black holes. The Sharma-Mittal and Rényi entropy contents of black holes have also been calculated and we saw that Rényi entropy leads to Bekenstein entropy. On the other hand, although Sharma-Mittal entropy is a two free parameters entropy measure [4], we found out that its final estimation of black hole entropy is mathematically similar to that of Tsallis entropy.

Next, applying the HDE hypothesis to the obtained Kaniadakis entropy, a new HDE model (KHDE) is derived. Our study shows that KHDE can model the current accelerated universe and furthermore, suitable transition from the matter dominated era to the current era is achievable by choosing proper values for the model parameters including $C^{2}$ and $\kappa$. We also used the Tsallis entropy (5) to construct a new Tsallis HDE model, in short NTHDE, and studied its cosmological outcomes. It has been found out that its general behavior is relatively like that of KHDE, and depending on the values of $\delta$ and $D^{2}$, different transition redshifts are achievable.

Finally, we addressed the models estimations of allowed ranges for the universe age, and found out that the age problem can be avoided in these models, depending on the values of the models parameters including $\left(\kappa, C^{2}\right)$ for KHDE, and $\left(\delta, D^{2}\right)$ for the second one. The validity of $\frac{1}{\frac{3 H^{2}}{8 \pi}} \leq \frac{1}{\frac{3 H^{2}}{8 \pi}+p_{\Lambda}}=\frac{\left(1+w_{\Lambda} \Omega_{\Lambda}\right)^{-1}}{\frac{3 H^{2}}{8 \pi}} \leq \frac{\left(1+w_{\Lambda} \Omega_{\Lambda}\right)_{z=0}^{-1}}{\frac{3 H^{2}}{8 \pi}}$, due to the facts that (i) $\dot{H}, p_{\Lambda}<0$, and (ii) $-p_{\Lambda}<\rho_{\Lambda}<\rho_{c}$ during the cosmic evolution, was the backbone of our estimations of the universe age.

Acknowledgements We are grateful to the anonymous referee for the valuable constructive comments. MKZ would like to thank Shahid Chamran university of Ahvaz, Iran for supporting this work.

Data Availability Statement This manuscript has no associated data or the data will not be deposited. [Authors' comment: All data included in the present work are available upon request by contacting with the corresponding author.]

Open Access This article is licensed under a Creative Commons Attribution 4.0 International License, which permits use, sharing, adaptation, distribution and reproduction in any medium or format, as long as you give appropriate credit to the original author(s) and the source, provide a link to the Creative Commons licence, and indicate if changes were made. The images or other third party material in this article are included in the article's Creative Commons licence, unless indicated otherwise in a credit line to the material. If material is not included in the article's Creative Commons licence and your intended use is not permitted by statutory regulation or exceeds the permitted use, you will need to obtain permission directly from the copyright holder. To view a copy of this licence, visit http://creativecomm ons.org/licenses/by/4.0/.

Funded by $\mathrm{SCOAP}^{3}$.

\section{References}

1. J.W. Gibbs, Statistical Mechanics, Mathematical physics (Charles Scribner's Sons, New York, 1902)

2. G. Kaniadakis, Phys. A 296, 405 (2001)

3. G. Kaniadakis, Phys. Rev. E 66, 056125 (2002)

4. M. Masi, Phys. Lett. A 338, 217 (2005)

5. H. Moradpour, Int. J. Theor. Phys. 55, 4176 (2016)

6. R.C. Nunes, E.M. Barboza Jr., E.M.C. Abreu, J.A. Neto, JCAP 08 , 051 (2016)

7. N. Komatsu, Eur. Phys. J. C 77, 229 (2017)

8. H. Moradpour, A. Bonilla, E.M.C. Abreu, J.A. Neto, Phys. Rev. D 96(12), 123504 (2017)

9. E. Abreu, M.C.J.A. Neto, A.C.R. Mendes, A. Bonilla, R.M. de Paula, EPL 124(3), 30005 (2018)

10. A. Bialas, W. Czyz, Euro. Phys. Lett. 83, 60009 (2018)

11. A. Belin, A. Maloney, S. Matsuura, J. High Energ. Phys. 2013, 50 (2013)

12. T.S. Biró, V.G. Czinner, Phys. Lett. B 726, 861 (2013)

13. V.G. Czinnera, H. Iguchia, Phys. Lett. B 752, 306 (2016)

14. V.G. Czinnera, H. Iguchia, Eur. Phys. J. C 77, 892 (2017)

15. S. Ghaffari, H. Moradpour, A.H. Ziaie, F. Asghariyan, F. Feleppa, M. Tavayef, Gen. Rel. Grav. 51(7), 93 (2019)

16. J. Sadeghi, M. Rostami, M.R. Alipour, Int. J. Mod. Phys. A 34(30), 1950182 (2019)

17. M. Tavayef, A. Sheykhi, K. Bamba, H. Moradpour, Phys. Lett. B 781, 195-200 (2018)

18. A. Sayahian Jahromi, S.A. Moosavi, H. Moradpour, J.P. Morais Graça, I.P. Lobo, I.G. Salako, A. Jawad, Phys. Lett. B 780, 21-24 (2018)

19. H. Moradpour, S.A. Moosavi, I.P. Lobo, J.P. Morais Graça, A. Jawad, I.G. Salako, Eur. Phys. J. C 78(10), 829 (2018)

20. H. Moradpour, A. Sheykhi, C. Corda, I.G. Salako, Phys. Lett. B 783, 82-85 (2018)

21. H. Moradpour, A.H. Ziaie, S. Ghaffari, F. Feleppa, Mon. Not. R. Astron. Soc. 488(1), L69-L74 (2019) 
22. H. Moradpour, C. Corda, A.H. Ziaie, S. Ghaffari, EPL 127(6), 60006 (2019)

23. J.D. Barrow, Phys. Letts. B 808, 135643 (2020)

24. S. Ghaffari, A.H. Ziaie, V.B. Bezerra, H. Moradpour, Mod. Phys. Lett. A 35(01), 1950341 (2019)

25. M. Srednicki, Phys. Rev. Lett. 71, 666 (1993)

26. S. Das, S. Shankaranarayanan, Class. Quant. Grav. 24, 5299 (2007)

27. D. Pavon, arXiv:2001.05716 [gr-qc] (2020)

28. M. Li, Phys. Lett. B 603, 1 (2004)

29. Y.S. Myung, Phys. Lett. B 652, 223 (2007)

30. S.A. Hayward, Class. Quantum Grav. 15, 3147 (1998)

31. S.A. Hayward, S. Mukohyana, M.C. Ashworth, Phys. Lett. A 256, 347 (1999)

32. D. Bak, S.J. Rey, Class. Quantum Grav. 17, 83 (2000)

33. R.G. Cai, S.P. Kim, J. High Energy Phys. 0502, 050 (2005)

34. M. Akbar, R.G. Cai, Phys. Rev. D 75, 084003 (2007)

35. R.G. Cai, L.M. Cao, Y.P. Hu, Class. Quantum. Grav. 26, 155018 (2009)

36. Q. Huang, H. Huang, J. Chen, L. Zhang, F. Tu, Class. Quant. Grav. 36(17), 175001 (2019)
37. M. Nauenberg, Phys. Rev. E 67, 036114 (2003)

38. L. G. Moyano, F. Baldovin, C. Tsallis, arXiv:cond-mat/0305091 (2003)

39. C. Tsallis, Phys. Rev. E 69, 038101 (2004)

40. M. Nauenberg, Phys. Rev. E 69, 038102 (2004)

41. W. Li, Q.A. Wang, L. Nivanen, A. le Mehaute, Eur. Phys. J. B 48, 95 (2005)

42. S. Abe, Phys. A 368, 430 (2006)

43. T.S. Biro, P. Van, Phys. Rev. E 83, 061147 (2011)

44. T.S. Biro, P. Van, Phys. Rev. E 84, 019902(E) (2011)

45. E. Abreu, M.C.J.A. Neto, A.C.R. Mendes, A. Bonilla, R.M. de Paula, EPL 124(3), 30003 (2018)

46. K. Mejrhit, S.E. Ennadifi, Phys. Lett. B 794, 24 (2019)

47. E.T. Jaynes, Am. J. Phys. 33, 391 (1965)

48. P.A.R. Ade et al., [Planck], Astron. Astrophys. 594, A13 (2016)

49. H.E. Bond, E.P. Nelan, D.A. VandenBerg, G.H. Schaefer, D. Harmer, Astrophys. J. Lett. 765, L12 (2013) 